\title{
Observed Properties of Dark Matter on Small Spatial Scales
}

\author{
Rosemary F.G. Wyse ${ }^{1}$ and Gerard Gilmore ${ }^{2}$ \\ ${ }^{1}$ Department of Physics \& Astronomy, Johns Hopkins University, Baltimore, MD 21218, USA \\ email: wyse@pha.jhu.edu \\ ${ }^{2}$ Institute of Astronomy, Cambridge, CB3 0HA, UK \\ email: gil@ast.cam.ac.uk
}

\begin{abstract}
The nature of dark matter is one of the outstanding questions of astrophysics. The internal motions of member stars reveal that the lowest luminosity galaxies in the Local Group are the most dark-matter dominated. New large datasets allow one to go further, and determine systematic properties of their dark matter haloes. We summarise recent results, emphasising the critical role of the dwarf spheroidal galaxies in understanding both dark matter and baryonic processes that shape galaxy evolution.
\end{abstract}

Keywords. dark matter, Local Group, galaxies: kinematics and dynamics

\section{Introduction}

The study of dark matter is best undertaken in systems that are the most darkmatter dominated, and for which the baryon content has had minimal effect on the dark matter halo. The dwarf spheroidal satellite galaxies of the Milky Way are such systems, with their high total dark-matter content being inferred some 25 years ago (Aaronson 1983) from their central stellar velocity dispersions (based on as few as three stars). Modern multi-object spectrographs on large telescopes make it possible now to acquire and analyse statistically significant samples of stars (many hundreds) across the face of these systems. This allows derivation of mass profiles.

Study of these smallest systems has the further advantage that it is on the smallest scales where the predictions of galaxy formation models with different types of dark matter diverge and are most easily discriminated (Ostriker \& Steinhardt 2003).

The dwarf spheroidal galaxies (dSph) are low surface-brightness, gas-poor systems, identified through star counts. They are the most common galaxy in the local Universe. The 'classical' dSph (see e.g. Gallagher \& Wyse 1994; Mateo 1998) were generally detected prior to 1990 , are at distances of $\sim 70 \mathrm{kpc}$ to $\sim 150 \mathrm{kpc}$, have typical total luminosity of $\sim 10^{7} \mathrm{~L}_{V, \odot}$, characteristic surface brightness of $\mu_{V} \sim 24 \mathrm{mag} / \mathrm{sq}$ arcsec and are extremely gas-poor. The central stellar velocity dispersion is $\sim 10 \mathrm{~km} / \mathrm{s}$, and combined with the characteristic radius of a few hundred parsec, this implies mass-to-light ratios in solar units in the range of $10 \lesssim M / L_{V} \lesssim 300$. The stars are metal-poor, with a typical mean $[\mathrm{Fe} / \mathrm{H}] \lesssim-1.5$ dex. All dSph contain old stars, and initiated star formation at early times, corresponding to a lookback time of $\gtrsim 12$ Gyr. More surprisingly, given the expectation of early supernovae-driven winds from these shallow potential wells (e.g. Dekel \& Silk 1986; see also Wyse \& Silk 1985 for a simpler derivation of the threshold escape velocity) most dSph contain stars of a very broad range of ages, and indeed the average dSph member star is of intermediate-age (e.g. Smecker-Hane et al. 1994; 
Hernandez, Gilmore \& Valls-Gabaud 2000). If there were (supernovae-driven?) outflows, the gas must have been re-accreted. $\dagger$

The dSph are then among the first systems to collapse and form stars, and have potential-well depths shallow enough for the baryonic content to be considerably affected by reionization of the Universe (a one-dimensional velocity dispersion of $10 \mathrm{~km} / \mathrm{s}$ corresponds to of order $\sim 10^{4} \mathrm{~K}$, approximately the ionization equilibrium temperature of hydrogen; see Efstathiou 1992; Bullock, Kravtsov \& Weinberg 2000). This potentialwell depth is also sufficiently shallow that, as noted above, internal effects - such as ionizing photons from massive stars, and energy and momentum injection from stellar winds and supernovae - can have a significant effect. Ram pressure, as the dwarf galaxy moves through the intergalactic medium (or through the gaseous halo of the Milky Way), can also cause gas to be removed from the dwarf. Thus the star formation histories and chemical evolutions of the dSph, which can be derived from observations such as deep colour-magnitude diagrams combined with spectroscopic elemental abundance measurements, can be used to constrain the physics assumed in theories of galaxy evolution that invoke significant 'feedback' on galactic scales to modify their stellar content e.g. Springel et al. (2005). 'Feedback' is not a free parameter.

As noted, these are the most dark-matter dominated galaxies. Analyses of large samples of internal velocities in galaxies over as wide a range of luminosity as possible will allow the quantification of trends in the inferred properties of the dark matter content with, e.g., galaxy luminosity or scale-length, and thereby place constraints on the type of dark matter (e.g. Dekel \& Silk 1986; Kormendy \& Freeman 2004; Zaritsky, Gonzalez \& Zabludoff 2006). Whether or not the dSph fit smoothly onto extrapolations of scaling relations seen for normal large galaxies is an interesting question, with implications for dark matter. The (model-dependent, at present) mass profile of individual dark haloes may be obtained from the stellar line-of-sight velocities, and compared to the predictions for different types of dark matter. It has long been known that the simplest variant of $\Lambda \mathrm{CDM}$ predicts too many dwarf-galaxy-mass dark haloes compared to the number of satellite galaxies (Moore et al. 1999; Klypin et al. 1999) and the derived mass functions and luminosity functions of the observed satellites are crucial in testing models, as are their detailed stellar populations.

The sample of known dSph satellites of the Milky Way has been recently increased by a factor of about two, primarily through the analysis of the uniform wide-field photometry of the stellar sky from the Sloan Digital Sky Survey. These new detections are of significantly lower luminosity, extending down to $\lesssim 10^{3} \mathrm{~L}_{\odot}$, and lower mean surface brightness, typically $\mu_{V} \sim 30 \mathrm{mag} / \mathrm{sq}$ arcsec (see Belokurov et al. 2006, Belokurov et al. 2007 and references therein.) Thus these galaxies overlap with star clusters in terms of total luminosity, and the question of what distinguishes star clusters from galaxies gains new relevance. Apart from the existence of a dark matter halo - essentially the definition of a 'galaxy' in the present paradigm - star clusters were known to have larger (stellar) phase space densities than galaxies, with the dSph having the largest phase space densities of galaxies, but some two orders of magnitude below the lowest phase space densities of star clusters (Walcher et al. 2005).

We here discuss the issue of what distinguishes star clusters from galaxies, using new radial-velocity data plus new photometric surveys and wide-field photometry of individual

$\dagger$ While outside the scope of this talk, there is a real lack of cosmologically consistent models of chemical evolution of the dSph that also are consistent with the individual star formation histories and do not invoke ad hoc outflows and inflows; see Silk, Wyse \& Shields (1987) for an early attempt. 


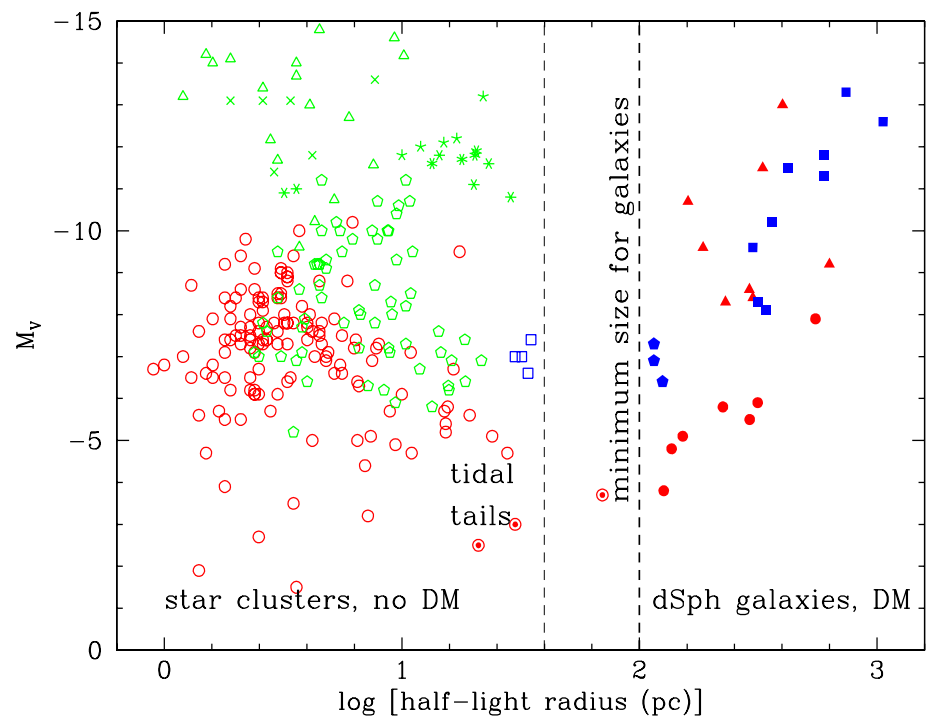

Figure 1. Absolute magnitude versus stellar half-light radius, for samples of star clusters (on the left of the dashed lines), including nuclear star clusters, young super-star-clusters, UCDs, old globular clusters, and on the right of the dashed lines, dwarf spheroidal galaxies of the Milky Way and of M31. This figure is modified from Fig. 1 of Gilmore et al. (2007), where full references are given.

systems. These data allow us to identify key characteristics of dark matter (see Gilmore et al. 2007 for a comprehensive discussion).

\section{Perspectives from New Data}

\subsection{Inferences from Photometry}

As noted above, the uniform, accurate and precise photometry from the Sloan Digital Sky Survey (particularly the coverage of DR5) has allowed discovery of many 'new' satellite galaxies and star clusters, through counts of faint stars and colour-magnitude matching. A very recent derivation of the satellite-galaxy luminosity function based on the detections in DR5, correcting for the calculated completeness and with assumed density laws for the satellite system, is given in Koposov et al. (2007). No published luminosity function based on semi-analytic prescriptions can provide a reasonable fit over the entire satellite luminosity range. A model including tidal stripping (Benson et al. 2002) can provide a good fit to the luminosity function of the faintest systems, but the faint galaxies in the model have predicted surface brightnesses many magnitudes brighter than those of the observed systems. This model also fails, with a shortfall of over an order of magnitude, for the luminosity function of bright satellites. It is clear that the models must be modified.

The new satellites further extend the overlap between star clusters and galaxies in terms of total luminosity. This is illustrated in Fig. 1, which is a plot of stellar half-light radius against total $\mathrm{V}$-band luminosity. The objects represented include a comprehensive variety of star clusters, from old galactic globular clusters through young super-star clusters, and also nuclear stars clusters and UCDs (or Ultra Compact Dwarf, which is probably not an apt description, discussed further below) in galaxy clusters (see Gilmore et al. 2007 for the references). The known dwarf spheroidal satellite galaxies of the Milky Way (excluding the Sagittarius dwarf) and of M31 are also represented. 
It is apparent that the characteristic stellar radius of star clusters is less than $30 \mathrm{pc}$, while the characteristic stellar radius of dSph is greater than 100pc. The 'gap', delineated by the dashed lines, is occupied by one object, the recently discovered system in Coma Berenices (Belukoruv et al. 2007). Deep imaging with Subaru reveals an extended, irregular structure; this, combined with the low estimated distance of $\sim 44 \mathrm{kpc}$, suggests tides may have affected its structure. Indeed the globular clusters with well-defined tidal tails lie just to the left of the 'gap', consistent with tidal effects being important in this size regime. Our hypothesis is that no systems in equilibrium should be in the 'gap'. Further, we interpret this figure as showing a real division between (stable) dark-matter dominated systems i.e. galaxies, in which the stellar scale-length is never below 100pc, and baryon-dominated star clusters, in which the scale-length is never above 30pc.

In our interpretation the UCDs (the asterisks in Fig. 1) are baryon-dominated star clusters, rather than distinct dark-matter dominated galaxies. This is consistent with the findings of Hilker et al. (2007) for bright UCDs in the Fornax cluster and of Evstigneeva et al. (2007) for bright UCDs in the Virgo cluster. The V-band mass-to-light ratios of the UCDs are generally in the range of 3-5 in solar units; this is higher than typical of globular clusters, but still consistent with a purely stellar system, due to the higher metallicity of the UCDs, typically above -1 dex (see these two papers for a full discussion including comparisons with a variety of spectral-synthesis models). A higher mass-to-light ratio $(\sim 9)$ was inferred for a somewhat less-luminous UCD in the Virgo cluster by Haşegan et al. (2005). Those authors suggest that such UCDs could be the remnant stellar nuclei of destroyed dwarf galaxies.

We conclude that all systems with (stellar) scale-length greater than $\sim 100 \mathrm{pc}$ have dark matter haloes, while there are no (stable) dark-matter dominated systems with scale length less than this. Only pure stellar systems have very small scale-lengths.

\subsection{Inferences from Stellar Kinematics}

Dwarf spheroidal galaxies are gas-poor, and in general the stars show no net rotation about the centre of the dSph. Constraints on the mass profiles are then obtained by analyses of the stellar (random) motions, in general the line-of-sight velocities. There are now statistically significant samples of stars with measured line-of-sight velocities, across the extent of the dSph on the sky - several hundred stars per galaxy - for most of the 'classical' dSph. These in general show flat or rising velocity-dispersion profiles, in contrast to the steadily declining profiles predicted for a mass-follows-light model - as seen in globular clusters e.g. $\omega$ Centauri, see Fig. 3 of van de Ven et al. (2006). This is compelling evidence for dark matter in the dSph.

\subsubsection{Derived Mass Profiles}

The most straightforward approach to determining the mass profile from the line-ofsight velocities is to use the Jeans equations to analyse the second moment of the velocity distribution in various bins in projected radius, i.e. the velocity dispersion profile. A full velocity distribution-function analysis would be better, but this requires very large samples to define the wings, and is significantly more complicated. Where both full distribution-function modelling and Jeans-equation modelling are available, they agree (e.g. compare Wu 2007 and Gilmore et al. 2007). However, the analysis of the stellar motions is complicated by the fact that, without proper motions, we measure only one component of the velocities. There is therefore a degeneracy between velocity-dispersion anisotropy and mass (see Binney \& Mamon 1982 for an early discussion).

The simplest assumption is that the velocity-dispersion tensor is isotropic. The derived mass profiles from Jeans-equation modelling, with assumed isotropic velocity-dispersion 


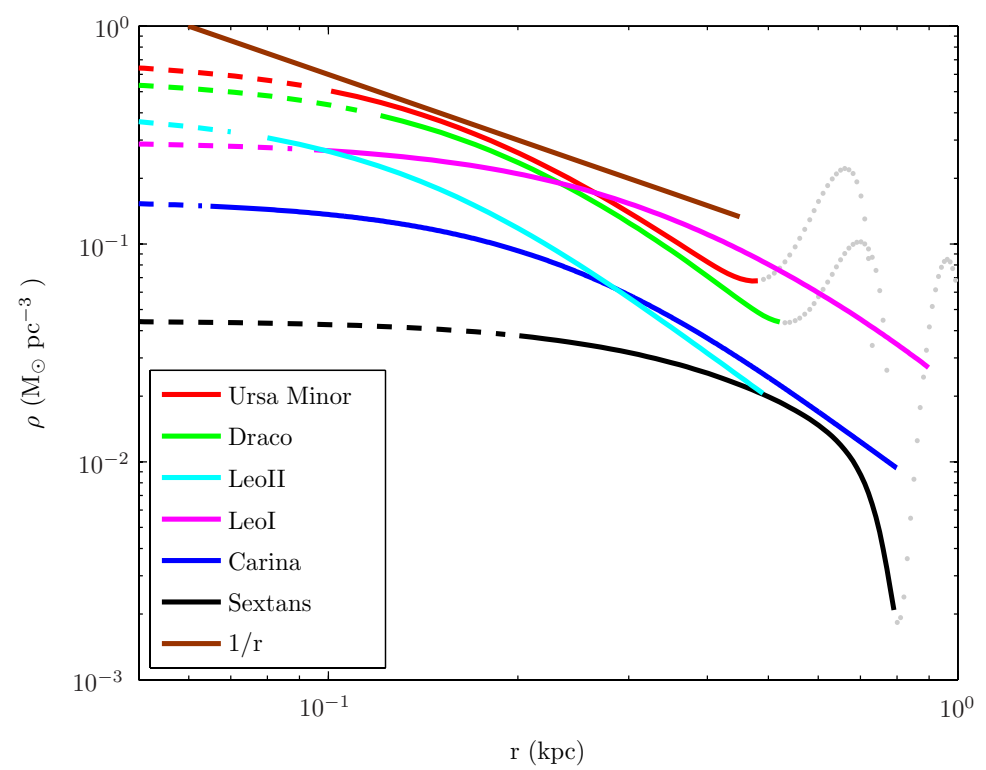

Figure 2. Derived mass density profiles from (isotropic) Jeans-equation analyses of the stellar velocity dispersion profiles of 6 dwarf spheroidal galaxies. Also shown is a $r^{-1}$ profile, to which $C D M$-mass profiles are predicted to asymptote. The modelling in each case is reliable out to $r \lesssim 500$ pc. This figure is taken from Gilmore et al. (2007)

tensor, is shown in Fig. 2, taken from Gilmore et al. (2007). Cold-dark-matter-dominated N-body simulations predict a cusp at the central regions; a recent high-resolution simulation finds that the mass density profile has a slope of -1.2 at $1 \%$ of the virial radius, and asymptotes to a slope of -1 (Diemand et al. 2005). This prediction is also shown in Fig. 2, and is clearly steeper than the derived profiles, which instead show a core. The model fits give a core radius of the mass distribution somehat larger than that of the light, as expected since gaseous baryons dissipate to form stars.

However, adopting an anisotropic velocity-dispersion tensor allows CDM-cusps to be fitted to the same data (Koch et al. 2007a). Happily, the mass-anisotropy can be broken for two of the dSph, using complementary independent physical arguments, and cored mass profiles are strongly favoured. Occam's razor then argues that cored mass profiles are favoured for all dSph. In the case of the UMi dSph, the persistence of an observed cold sub-system is not compatible with a cusp, since the strong gradients of the cusp would lead to disruption of the sub-system (Kleyna et al. 2003). In the case of the Fornax $\mathrm{dSph}$, its globular cluster system would have been expected to have long ago spiralled into the galaxy center, by dynamical friction, if the mass density profile were cusped at the centre, while a cored mass profile allows for survival of the globulars (Hernandez \& Gilmore 1998, Goerdt et al. 2006).

It is difficult to extend this analysis to the lowest luminosity systems, since there simply are very few stars accessible with $8 \mathrm{~m}-10 \mathrm{~m}$ class telescopes.

The conclusion is then that cored mass density profiles are preferred. It is also interesting that there is a fairly narrow range of derived mean mass densities, and that this typical value is rather low.

\subsubsection{Integrated Masses}

Mateo (1998) synthesised early results by estimating 'total' masses for dSph from their central velocity dispersions and half-light radii. He found that a constant mass 


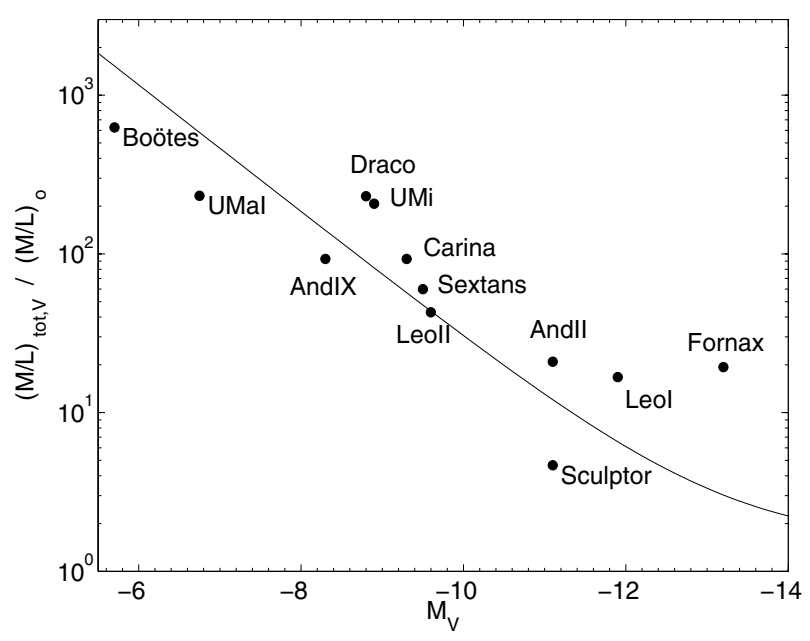

Figure 3. Mass-to-light ratios from the enclosed mass within the limit of stellar kinematic data for the profiles of Fig. 2, supplemented with galaxies for which more limited kinematic data are available, against absolute magnitude. There is a remarkably small scatter about a line of constant mass, given by the smooth curve. This figure is taken from Gilmore et al. (2007)

$\left(\sim 10^{7} \mathrm{M}_{\odot}\right)$ was a reasonable description of the data, albeit with a large scatter. It is now possible to improve on his analysis in two ways: first, by estimating masses through integration of the mass profiles from velocity data beyond the central regions of the galaxies, and second, by extending, down another 3 magnitudes, the luminosity range over which mass estimates can be made from central dispersions. The result is seen in Figure 3 (taken from Gilmore et al. 2007). Here the curve of constant mass represents $4 \times 10^{7} \mathrm{M}_{\odot}$, and the scatter is remarkably small. There is an apparently characteristic (lower) mass to the dark matter haloes that host galaxies.

There are hints from even lower luminosity galaxies, for which only central velocity dispersions are available, that their masses may be lower (Simon \& Geha 2007) but the member stars are so few that the velocity dispersions are poorly defined, and any exclusion of 'outliers' of course reduces the dispersion and reduces the derived mass.

\section{Stellar Mass Function}

It should be noted that while the (high) dark-matter content of dSph galaxies contrasts with that of globular clusters (none), the stellar component of the dSphs is consistent with a stellar Initial Mass Function indistinguishable from that of the globular clusters. The case of the dSph in Ursa Minor is the most straightforward to analyse, since its stellar population is old and metal-poor, of similar age and metallicity to the halo globular clusters of the Milky Way. Direct star counts with the Hubble Space Telescope demonstrate no differences between the low-mass stellar luminosity function of the UMi dSph and that of M92 or M15, two globular clusters (see Fig. 4, taken from Wyse et al. 2002). The initial mass function of massive stars may be constrained by the nucleosynthetic signature that persists in the elemental abundances of the long-lived, low-mass stars they enriched (e.g. review of Wyse 1998). The elemental abundance data for stars in $\mathrm{dSph}$ are consistent with a normal massive-star mass function, plus the (usually extended) star formation history inferred from the colour-magnitude diagram (e.g. Tolstoy et al. 2003; Koch et al. 2007b). This 'normal' IMF is of course equal to that seen in the 


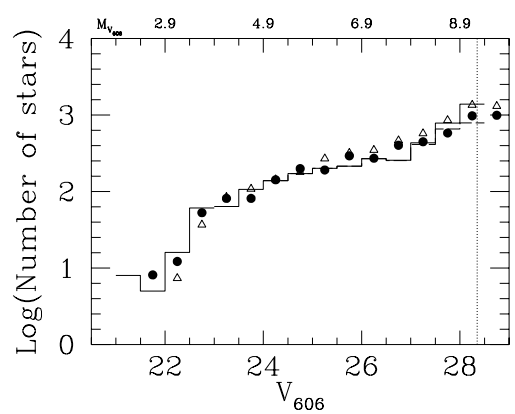

(a)

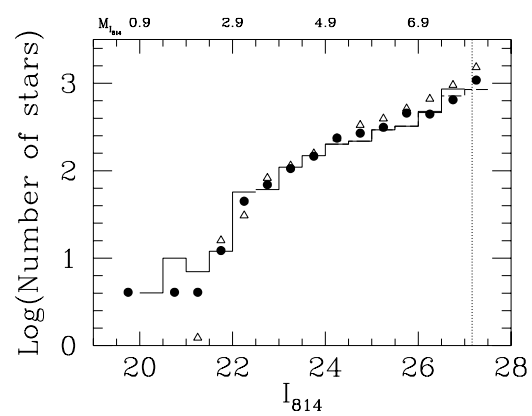

(b)

Figure 4. Based on figures in Wyse et al. (2002). Comparisons between the completeness-corrected Ursa Minor luminosity functions (histograms; $50 \%$ completeness indicated by the vertical dotted line) in the V-band (a) and the I-band (b) and the same for M92 (filled circles) and M15 (open triangles) (both taken from Piotto et al. 1997, renormalized and shifted to the same distance as the Ursa Minor dSph). The luminosity functions for the globular clusters and the dwarf spheroidal galaxy are indistinguishable.

solar neighbourhood and inferred for the galactic bulge: the stellar IMF is remarkably robust, apparently invariant over $12 \mathrm{Gyr}$ and a wide range of metallicities and epochs.

Thus the stellar masses of dSph and globular clusters are quite comparable; their dark matter contents - and stellar scale lengths - are what distinguishes them.

\section{Concluding Remarks}

There appears to be a minimum intrinsic scale length - of greater than $100 \mathrm{pc}-$ of galaxies and their associated dark matter haloes. The density profiles of the dark matter haloes are cored, not cusped, and have a low mean mass density of around $0.1 \mathrm{M}_{\odot} \mathrm{pc}^{-3}$, or $\sim 5 \mathrm{GeV} / \mathrm{cc}$, only around a factor of ten higher than the local dark matter density around the position of the Sun. The combination of characteristic scale with characteristic density leads to the expectation of a constant mass, and this is indeed what is found.

Thus if the dark matter particle is massive - and candidates more massive than $100 \mathrm{GeV}$ are serious candidates - then it must be extremely dilute to provide the central density cores. The characteristic length scale and mass are suggestive of a characteristic scale in the primordial power spectrum; the suppression of small-scale power that this would imply could perhaps naturally solve the well-known 'missing satellite' problem in CDM models, together with removing the prediction of central cusps. Non-CDM candidates (e.g. sterile neutrinos) need to considered seriously.

The possible effects of astrophysics in setting the stellar content of dSph cannot be ignored in interpreting the results presented here. Models that appeal to internal and/or external 'feedback' must derive the characteristic mass self-consistently, and fit the surface brightnesses, chemical elemental abundances, star formation histories and luminosity function. Happily there are now good observational constraints on all these aspects. The field stellar halo and thick disk must also be included in the models, since stellar debris from disrupted satellite galaxies will most likely be deposited there. The old age of the stars in these components contrasts with the typical intermediate-age population of surviving satellites and is a major constraint (Unavane, Wyse \& Gilmore 1996).

In the near future, we will have improved stellar kinematic data for the Carina dSph (new approved VLT programme; PI: Gilmore) to which to apply a full velocity 
distribution-function analysis. Improved proper motions for the dSph - and new proper motions for the more recently discovered systems - are needed to constrain their orbits and understand possible environmental effects on their evolution (HST application, then GAIA). The field is moving quickly.

\section{Acknowledgements}

RFGW acknowledges the Aspen Center for Physics, where this paper was written, for a stimulating environment. We thank Jon Davies for his flexibility faced with our substitution as speakers. We also acknowledge our collaborators in our dSph project: Mark Wilkinson, Jan Kleyna, Andreas Koch, Wyn Evans and Eva Grebel.

\section{References}

Aaronson, M. 1983, ApJL 266, L11

Belokurov, V. et al. 2006, ApJL 642, L137

Belokurov, V. et al. 2007, ApJ 654, 897

Benson, A. et al. 2002, ApJ 654, 897

Binney, J. \& Mamon, G.A. 1982, MNRAS 200, 361

Bullock, J., Kravtsov, A. \& Weinberg, D. 2000, ApJ 539, 517

Dekel, A. \& Silk, J. 1986, ApJ 303, 39

Diemand, J. et al. 2005, MNRAS 364, 665

Efstathiou, G. P. 1992, MNRAS 256, 43P

Evstigneeva, E. et al. 2007, AJ 133, 1722

Gallagher, J. S., III \& Wyse, R. F. G. 1994, PASP 106, 1225

Gilmore, G., Wilkinson, M. I., Wyse, R. F. G., Kleyna, J. T., Koch, A., Evans, N. W. \& Grebel, E. $2007, A p J 663,948$

Goerdt, T., Moore, B., Read, J. I., Stadel, J. \& Zemp, M. 2006, MNRAS 3681073

Haşegan, M. et al. 2005, ApJ 627, 203

Hernandez, X. \& Gilmore, G. 1998, MNRAS 297, 517

Hernandez, X., Gilmore, G. \& Valls-Gabaud, D. 2000, MNRAS 317, 831

Hilker, M. et al. 2007, A\&SA 463, 119

Kleyna J. T., Wilkinson M. I., Gilmore G. \& Evans N. W. 2003, ApJL 588, L21

Klypin, A. et al. 1999, ApJ 552, 82

Koch, A., Kleyna J. T., Wilkinson M. I., Grebel, E., Gilmore G., Evans N. W., Wyse, R. F. G. \& Harbeck, D. 2007a, AJ 134, 566

Koch, A., Grebel, E., Gilmore G., Wyse, R. F. G., Kleyna J. T., Harbeck, D., Wilkinson M. I. \& Evans N. W. 2007b, $A J$ submitted

Koposov, S. et al. 2007, submitted to ApJ (arXiv0706.2687)

Kormendy, J. \& Freeman, K. C. 2004, in: S. Ryder et al. (eds.), IAU Symposium 220 p. 377

Mateo, M. 1998, ARAA 36, 435

Moore, B. et al. 1999, ApJL 524, L19

Ostriker, J. P. \& Steinhardt, P. 2003, Science 300, 1909

Piotto, G., Cool, A. \& King, I. 1997, AJ 113, 1345

Silk, J. I., Wyse, R. F. G. \& Shields, G. 1987, ApJL 322, L59

Simon, J. \& Geha, M. 2007, submitted to ApJ (arXiv0706.0516)

Smecker-Hane, T. et al. 1994, AJ 108, 507

Springel, V. et al. 2005, Nature 435, 629

Tolstoy, E. et al. 2003, AJ 125, 707

Unavane, M., Wyse, R. F. G. \& Gilmore, G. 1996, MNRAS 278, 727

van de Ven, G. et al. 2006, A\&\&A 445, 513

Walcher, C. J. et al. 2005, ApJ 618, 237

Wu, X. 2007. submitted to ApJ (astro-ph/0702233) 
Wyse, R. F. G. 1998, in: G. Gilmore \& D. Howell (eds.), ASP Conference Series 142, 89 Wyse, R. F. G. \& Silk, J. 1985, ApJL 296, L1

Wyse, R. F. G. et al. 2002, New Astr 7, 395

Zaritsky, D., Gonzalez, A. \& Zabludoff, A. 2006, ApJ 638, 725 